Real Analysis Exchange

Vol. 24(2), 1998/9, pp. 867-872

Vasile Ene, Ovidius University Constanţa, Romania

Current address: 23 August 8717, Jud. Constanţa, Romania

e-mail: ene@s23aug.sfos.ro or ene@univ-ovidius.ro

\title{
ON AN IMPROVEMENT OF THE HAKE THEOREM
}

\begin{abstract}
The well-known Hake Theorem asserts that if a function $f$ is Denjoy* integrable then it is also Perron integrable, and the two integrals are equal. In [3] we introduced a very strong Perron integration $\left(\mathcal{P}_{1,1}\right)$ and proved the corresponding Hake-type theorem, using the VitaliCarathéodory Theorem. In this paper we give a new, less technical proof of this result, using essentially Lusin's Theorem.
\end{abstract}

\section{Introduction}

The well-known Hake Theorem asserts that if a function $f$ is Denjoy* integrable then it is also Perron integrable, and the two integrals are equal. In fact these two integrals are equivalent (see the Hake-Alexandroff-Looman Theorem), and there are many definitions of Perron-type integrals that are equivalent to the Denjoy* integral. In [3, Corollary 5.9.1], we made a study of many (at least 108) of these equivalences. One of the strongest Perron type definition is that of Skljarenko, where the major and minor functions are $A C^{*} G$ and continuous. Using the Tolstoff-Zahorski Theorem we showed that in addition, the major and minor functions have finite or infinite derivatives at each point, obtaining the $\left(\mathcal{P}_{1,1}\right)$-integral. To prove that the $\mathcal{D}^{*}$-integrability implies the $\left(\mathcal{P}_{1,1}\right)$-integrability (i.e., a Hake type theorem) we used essentially the Vitali-Carathéodory Theorem $[11$, p. 166]. In the present paper we give a different, less technical proof of this result, using essentially Lusin's Theorem $[13$, p. 72$]$. Both proofs use different techniques from that of Skljarenko. We conclude the paper with some comments and a question related to the subject.

\footnotetext{
Key Words: $A C^{*} G$, the Perron integral, the Kurzweil-Henstock integral

Mathematical Reviews subject classification: 26A39, 26A42, 26A46

Received by the editors September 21, 1998

*The author died on November 11, 1998; see Real Anal. Exch. 241 (1998/99), 3.
} 


\section{Preliminaries}

For the definitions of $V B, V B^{*}, A C, A C^{*}, A C^{*} G$ see [13]. We denote by $\mathcal{P}(E)=\{X: X \subseteq E\}$ whenever $E \subseteq \mathbb{R}$. By $\mathcal{O}(F ; X)$ we mean the oscillation of the function $F$ on the set $X$. We denote by $m(X)$ the Lebesgue measure of the Lebesgue measurable set $X$.

Definition 1. Let $F:[a, b] \mapsto \mathbb{R}$, and let $\mathrm{P}$ be a closed subset of $[\mathrm{a}, \mathrm{b}]$, $c=\inf (P), d=\sup (P)$. Let $F_{P}:[c, d] \rightarrow \mathbb{R}$ be defined as follows: $F_{P}(x)=$ $F(x), x \in P$ and $F_{P}$ is linear on each $\left[c_{k}, d_{k}\right]$, where $\left\{\left(c_{k}, d_{k}\right)\right\}_{k \geq 1}$ are the intervals contiguous to $P$.

Definition 2. [3, p. 174]. Let $f:[a, b] \rightarrow \overline{\mathbb{R}}$. We define the following classes of major and minor functions and the corresponding $\left(\mathcal{P}_{1,1}\right)$-integral:

- $\overline{\mathcal{M}}_{1}(f)=\left\{M:[a, b] \rightarrow \mathbb{R}: M(a)=0, M \in A C^{*} G ; M^{\prime}(x)\right.$ exists (finite or infinite); $\left.f(x) \leq M^{\prime}(x) \neq-\infty\right\}$;

- $\underline{\mathcal{M}}_{1}(f)=\left\{m:[a, b] \rightarrow \mathbb{R}:-m \in \overline{\mathcal{M}}_{1}(-f)\right\}$.

- If $\overline{\mathcal{M}}_{1}(f) \neq \emptyset$ then we denote by $\bar{I}_{1}(b)$ the lower bound of all $M(b)$, $M \in \overline{\mathcal{M}}_{1}(f)$. If $\underline{\mathcal{M}}_{1}(f) \neq \emptyset$ then we denote by $\underline{I}_{1}(b)$ the upper bound of all $m(b), m \in \underline{\mathcal{M}}_{1}(f)$.

- $f$ is said to have a $\left(\mathcal{P}_{1,1}\right)$-integral on $[a, b]$, if $\overline{\mathcal{M}}_{1}(f) \times \underline{\mathcal{M}}_{1}(f) \neq \emptyset$ and $\bar{I}_{1}(b)=\underline{I}_{1}(b)=\left(\mathcal{P}_{1,1}\right) \int_{a}^{b} f(t) d t$.

Lemma A. [3, Lemma 5.8.2]. Let $F, H, G:[a, b] \rightarrow \mathbb{R}$, and let $H(x)=$ $\mathcal{O}(F ;[a, b])-\mathcal{O}(F ;[x, b])+\mathcal{O}(G ;[a, x]), G=F+H$. If $F \in A C^{*} G$ on $[a, b]$ then: $H(a)=0$ and $H(b)=2 \cdot \mathcal{O}(F ;[a, b]) ; H$ is increasing and $A C$ on $[a, b]$; $G \in A C^{*} G$ and $G(a) \leq G(x) \leq G(b)$ on $[a, b]$.

Lemma B. [3, Theorem 2.11.1, (xviii)]. Let $F:[a, b] \rightarrow \mathbb{R}$, and let $P$ be a subset of $[a, b], c=\inf (P) d=\sup (P) . F \in A C$ on $\bar{P}$ if and only if $F_{\bar{P}} \in A C$ on $[c, d]$;

Lemma C. Let $F:[a, b] \rightarrow \mathbb{R}, F \in A C$, and let $V:[a, b] \rightarrow \mathbb{R}, V(x)=$ $V(F ;[a, x])$ (here $V(f ; X)$ stands for the variation of $F$ on the set $X)$. Then $V \in A C$ on $[a, b]$.

Proof. This follows by definitions.

Lemma D. [3, Lemma 5.8.3]. Let $\left\{r_{k}\right\}_{k}$ be a sequence of positive numbers such that $\sum_{k=1}^{\infty} r_{k}=r<+\infty$. Let $F_{k}:[a, b] \rightarrow\left[0, r_{k}\right]$ such that $F_{k}$ is increasing and $A C$ on $[a, b]$. Let $F:[a, b] \rightarrow \mathbb{R}, F(x)=\sum_{k=1}^{\infty} F_{k}(x)$. Then: $F(a)=0$ and $F(b)<r ; F$ is increasing and $A C$ on $[a, b]$. 


\section{A Hake Type Theorem}

Lemma 1. Let $F:[a, b] \rightarrow \mathbb{R}, F \in A C^{*} G$ on $[a, b]$. Let $P$ be a closed subset of $[a, b]$ such that $F \in A C^{*}$ on $P$. Then for $\epsilon>0$, there is a $\delta>0$ such that for every open set $E$ with $m(E)<\delta$, there exists a function $F^{E}:[a, b] \rightarrow[0,+\infty)$ having the following properties:

a) $F^{E}(a)=0, F^{E}(b)<\epsilon$;

b) $F^{E}$ is $A C$ and increasing on $[a, b]$;

c) $\underline{D}\left(F+F^{E}\right)(x) \geq 0$ for all $x \in P \cap E \neq \emptyset$;

d) For some $E$ we have that $\underline{D}\left(F+F^{E}\right)$ is bounded below on $P$.

Proof. Let $Q=\{a, b\} \cup P, V:[a, b] \rightarrow \mathbb{R}, V(x)=V\left(F_{Q} ;[a, x]\right)$. By Lemma B and Lemma $\mathrm{C}$, the function $V$ belongs to $A C$ on $[a, b]$. Let $\left\{\left(a_{i}, b i\right)\right\}_{i=1}^{\infty}$ (we suppose the infinite case, otherwise the situation is easy) be the intervals contiguous to $Q$. Let $H_{i}:[a, b] \rightarrow[0,+\infty)$,

$$
H_{i}(x)= \begin{cases}0 & \text { if } x \in\left[a, a_{i}\right] \\ \mathcal{O}\left(F ;\left[a_{i}, b_{i}\right]\right)-\mathcal{O}\left(F ;\left[x, b_{i}\right]\right)+\mathcal{O}\left(F ;\left[a_{i}, x\right]\right) & \text { if } x \in\left[a_{i}, b_{i}\right] \\ 2 \cdot \mathcal{O}\left(F ;\left[a_{i}, b_{i}\right]\right) & \text { if } x \in\left[b_{i}, b\right]\end{cases}
$$

By Lemma A, each $H_{i}$ is $A C$ and increasing on $[a, b]$. Since $V(b)+\sum_{i=1}^{\infty} H_{i}(b)$ $\neq+\infty$ (because $F$ is $V B^{*}$ on $P$, so on $Q$ ), by Lemma $\mathrm{D}$, the function $G$ : $[a, b] \rightarrow \mathbb{R}, G(x)=V(x)+\sum_{i=1}^{\infty} H_{i}(x)$ is increasing and $A C$ on $[a, b]$. Hence $G^{\prime}$ exists and is finite a.e. on $[a, b], G^{\prime}(x) \geq 0$ a.e. on $[a, b], G^{\prime}$ is Lebesgue integrable on $[a, b]$, and $G(x)=(\mathcal{L}) \int_{a}^{x} G^{\prime}(t) d t$ (see [11, p. 255] or [3, p. 62]). For $\epsilon>0$, let $\delta>0$ be such that $\int_{S} G^{\prime}(t) d t<\epsilon$, whenever $S$ is a Lebesgue measurable subset of $[a, b]$ with $m(S)<\delta$ (see [11, p. 148]. Let $E$ be an open set with $m(E)<\delta, E \cap P \neq \emptyset$, and let $F^{E}:[a, b] \rightarrow \mathbb{R}, F^{E}(x)=$ $(\mathcal{L}) \int_{[a, x] \cap E} G^{\prime}(t) d t$. Clearly we have a) and b).

c) Fix some $x_{o} \in E \cap P$, and let $x>x_{o}$ such that $\left[x_{o}, x\right] \subset E \cap[a, b]$. Then

$$
F^{E}(t)-F^{E}\left(x_{o}\right)=(\mathcal{L}) \int_{\left[x_{o}, t\right]} G^{\prime}(z) d z, \quad(\forall) t \in\left[x_{o}, x\right] .
$$

Since $\left(F^{E}\right)^{\prime}(t)=G^{\prime}(t)$ a.e. on $\left[x_{o}, x\right]$, and $F^{E}, G \in A C$ on $[a, b]$, it follows that $F^{E}-G$ is constant on $\left[x_{o}, x\right]$. For $x \in P$ we have

$$
\left(F+F^{E}\right)(x)-\left(F+F^{E}\right)\left(x_{o}\right)=F(x)-F\left(x_{o}\right)+G(x)-G\left(x_{o}\right) \geq
$$




$$
\geq F(x)-F\left(x_{o}\right)+V(x)-V\left(x_{o}\right) \geq 0 .
$$

If $x \in\left(a_{i}, b_{i}\right)$ then from above, $\left(F+F^{E}\right)\left(a_{i}\right)-\left(F+F^{E}\right)\left(x_{o}\right) \geq 0$ and

$$
\begin{aligned}
\left(F+F^{E}\right)(x) & -\left(F+F^{E}\right)\left(a_{i}\right)=F(x)-F\left(a_{i}\right)+G(x)-G\left(a_{i}\right) \geq \\
& \geq F(x)-F\left(a_{i}\right)+H_{i}(x)-H_{i}\left(a_{i}\right) \geq 0
\end{aligned}
$$

(see the last part of Lemma A). Thus $\left(F+F^{E}\right)(x)-\left(F+F^{E}\right)\left(x_{o}\right) \geq 0$. Similarly, we obtain that $\left(F+F^{E}\right)\left(x_{o}\right)-\left(F+F^{E}\right)(x) \geq 0$ for $x<x_{o}$ and $\left[x, x_{o}\right] \subset E \cap[a, b]$ (in the computations we shall use $b_{i}$ instead of $a_{i}$ ). Therefore $\underline{D}\left(F+F^{E}\right)\left(x_{o}\right) \geq 0$.

d) Since $F \in A C^{*} G$ on $[a, b]$, it follows that $F^{\prime}(x)$ exists and is finite a.e. on $P$. But $F^{\prime}$ is also Lebesgue measurable on $P$. Thus by Lusin's Theorem (see $[13$, p. 72$]$ ), there exists a closed subset $P_{o}$ of $P$, such that $\left(F^{\prime}\right)_{\mid P_{o}}$ is continuous and $m\left(P \backslash P_{o}\right)<\delta / 2$. Hence there exists an open set $E$ such that $\left(P \backslash P_{o}\right) \subset E$ and $m(E)<\delta$. It follows that $\left(F^{\prime}\right)_{\mid P \backslash E}$ is bounded, and by b) and c) we have that $\underline{D}\left(F+F^{E}\right)$ is bounded below on $P$.

Theorem 1. [3, Corollary 5.8.1]. Let $F:[a, b] \rightarrow \mathbb{R}, F \in A C^{*} G$ on $[a, b]$, and let $r>0$. Then there exists $H:[a, b] \rightarrow \mathbb{R}$ such that:

(i) $H(a)=0$ and $H(b)<r$;

(ii) $H$ is increasing and $A C$ on $[a, b]$;

(iii) $G=F+H$ is $A C^{*} G$ and $\underline{D} G(x) \neq-\infty$ on $[a, b]$.

Proof. Since $F \in A C^{*} G$ on $[a, b]$, it follows that there exists a sequence $\left\{E_{n}\right\}_{n}$ of closed subsets of $[a, b]$ that cover $[a, b]$, such that $F$ is $A C^{*}$ on each $E_{n}$. By Lemma 1, for each positive integer $n$, there exists $h_{n}:[a, b] \rightarrow\left[0, \frac{r}{2^{n+1}}\right)$, such that $\underline{D}\left(F+h_{n}\right)(x) \neq-\infty$ for $x \in E_{n}$, and $h_{n}$ is $A C$ and increasing on $[a, b]$. Let $H:[a, b] \rightarrow \mathbb{R}, H(x)=\sum_{n=1}^{\infty} h_{n}(x)$. Clearly we have (i), and by Lemma D, we also have (ii) and (iii).

Theorem 2. [3, Theorem 5.8.1]. Let $f:[a, b] \rightarrow \overline{\mathbb{R}}$. If $f$ is $\mathcal{D}^{*}$ - integrable on $[a, b]$ then $f$ is $\left(\mathcal{P}_{1,1}\right)$-integrable on $[a, b]$ and the two integrals are equal.

Proof. The proof follows by Theorem 1 and the Tolstoff-Zahorski Theorem (see for example [3, Theorem 2.14.6]).

Remark 1. Special cases of Theorem 2 and Theorem 3 have been studied by many authors, from different points of view, using the constructive theory of Denjoy ([11, Ch. XVI, §8]), or the descriptive theory of Denjoy ([13], [14], [9], [3]), or the Kurzweil-Henstock theory ([4], [5], [15]), or combining the descriptive theory of Denjoy with the Kurzweil-Henstock theory ([10]). 


\section{Some Comments and Remarks}

In [2, Corollary 1, (i), (vii)] and [3, Corollary 2.27.1, (i), (vii)] we have proved, without using the Kurzweil-Henstock theory, the following result (two years later, B. Bongiorno, L. Di Piazza, and V. Skvortsov gave another proof, using the Kurzweil-Henstock theory [1, Theorem 4]):

Theorem A. A function $F:[a, b] \rightarrow \mathbb{R}$ is $A C^{*} G$ on $[a, b]$ if and only if $F$ is $Y_{D^{\circ}}$ on $[a, b]$.

Remark 2. Condition $Y_{D^{\circ}}$ has been introduced by Jarnik and Kurzweil, without naming it, in [6, (3.16) on p. 655]; later it has been used by several authors, but with different names: the strong Lusin condition, short $S L C$ in [8, p. 557], well-behaved in [7, p. 124], $A C_{*}$ in [12, p. 115], $Y_{D_{o}}$ in [2, p. 503], $[3$, p. 89], absolute continuity of the variational measure with respect to the Lebesgue measure in [1], etc.

From Theorem A we easily obtain the following theorem:

Theorem B. [3, Theorem 5.12.1, (i), (iv)]. $f:[a, b] \rightarrow \mathbb{R}$ is $\mathcal{D}^{*}$-integrable on $[a, b]$ if and only if $F$ is $(K H)$-integrable (Kurzweil-Henstock integrable) on $[a, b]$ and the two integrals are equal.

Using Theorem B (or only Theorem A and the facts that $F \in Y_{D^{\circ}}$ on $[a, b]$, and $F^{\prime}(x)=f(x)$ a.e. on $[a, b]$ for $\left.F(x)=(K H) \int_{a}^{x} f(t) d t, x \in[a, b]\right)$ together with Theorem 2, we obtain:

Theorem 3. If $f \in(K H)$-integrable on $[a, b]$ then $f$ is $\left(\mathcal{P}_{1,1}\right)$-integrable on $[a, b]$ and the two integrals are equal.

Question. In [15, Theorem 2], using the Kurzweil-Henstock theory, Skvortsov constructed some continuous major and minor functions $M, m$ for a $K H$ integrable function. Is it possible to show that $M$ and $m$ are also major respectively minor functions for the $\left(\mathcal{P}_{1,1}\right)$-integral?

\section{References}

[1] B. Bongiorno, L. Di Piazza, and V. Skvortsov, A new full descriptive characterization of Denjoy-Perron integral, Real Analysis Exchange 21 (1995/6), no. 2, 656-663.

[2] V. Ene, Characterization of $A C^{*} G \cap \mathcal{C}, \underline{A C^{*}} \cap \mathcal{C}_{i}, A C$ and $\underline{A C}$ functions, Real Analysis Exchange 19 (1993/4), no. 2, 491-510. 
[3] V. Ene, Real functions - current topics, Lect. Notes in Math., vol. 1603, Springer-Verlag, 1995.

[4] R. Henstock, Theory of integration, Butterworth London, 1963.

[5] R. Henstock, Majorants in variational integration, Canad. J. Math. 18 (1966), 49-74.

[6] J. Jarník and J. Kurzweil, A general form of the product integral and linear ordinary differential equations, Czech. Math. J. 37 (1987), no. 112, 642-659.

[7] J. Kurzweil and J. Jarnik, Equiintegrability and controlled convergence of Perron-type integrable functions, Real Analysis Exchange 17 (1991/2), no. $1,110-140$.

[8] P. Y. Lee, On ACG* functions, Real Analysis Exchange 15 (1989/90), no. $2,754-760$.

[9] G. Liu, P. Y. Lee, and P. S. Bullen, A note on major and minor functions for the Perron integral, Real Analysis Exchange 20 (1994/5), no. 1, 336339 .

[10] S. Lu, On the construction of major and minor functions, Journal of Math. Study 27 (1994), no. 1, 121-126.

[11] I. P. Natanson, Theory of functions of a real variable, 2nd. rev. ed., Ungar, New York, 1961.

[12] W. F. Pfeffer, The Riemann approach to integration, Cambrige Univ. Press, New York, 1993.

[13] S. Saks, Theory of the integral, 2nd. rev. ed., vol. PWN, Monografie Matematyczne, Warsaw, 1937.

[14] V. A. Skljarenko, Integration by parts in the SCP Burkill integral, Math. USSR Sbornik 40 (1981), 567-583.

[15] V. Skvortsov, Continuity of $\delta$-variation and construction of continuous major and minor functions for the Perron integral, Real Analysis Exchange 21 (1995/6), no. 1, 270-277. 